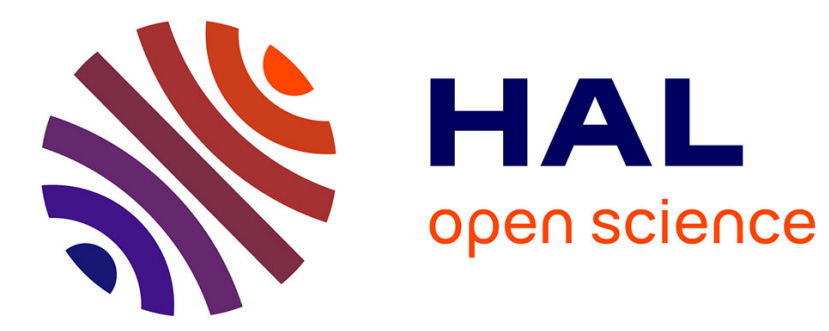

\title{
Consistance d'un estimateur de minimum de variance étendue
}

\author{
Joseph Rynkiewicz
}

\section{To cite this version:}

Joseph Rynkiewicz. Consistance d'un estimateur de minimum de variance étendue. Comptes rendus de l'Académie des sciences. Série I, Mathématique, 2005, 341, pp.133-136. hal-00258115

\section{HAL Id: hal-00258115 \\ https://hal.science/hal-00258115}

Submitted on 21 Feb 2008

HAL is a multi-disciplinary open access archive for the deposit and dissemination of scientific research documents, whether they are published or not. The documents may come from teaching and research institutions in France or abroad, or from public or private research centers.
L'archive ouverte pluridisciplinaire HAL, est destinée au dépôt et à la diffusion de documents scientifiques de niveau recherche, publiés ou non, émanant des établissements d'enseignement et de recherche français ou étrangers, des laboratoires publics ou privés. 


\title{
Consistance d'un estimateur de minimum de variance étendue
}

\author{
Consistency of a least extended variance estimator
}

\author{
Joseph Rynkiewicz ${ }^{\text {a }}$ \\ a SAMOS/MATISSE, Université de ParisI, 72 rue Regnault 75013 Paris, France, Tél. et Fax : 01-44-07-87-05
}

\begin{abstract}
We consider a generalization of the criterion minimized by the K-means algorithm, where a neighborhood structure is used in the calculus of the variance. Such tool is used, for example with Kohonen maps, to measure the quality of the quantification preserving the neighborhood relationships. If we assume that the parameter vector is in a compact Euclidean space and all it components are separated by a minimal distance, we show the strong consistency of the set of parameters almost realizing the minimum of the empirical extended variance. To cite this article:
\end{abstract}

\section{Résumé}

On considère une généralisation du critère minimisé par l'algorithme des K-moyennes [K-means], où une structure de voisinage est introduit dans le calcul de la variance. Un tel outil est utilisé, par exemple avec des cartes de Kohonen, pour mesurer la qualité de la quantification respectant les structures de voisinage. Si on suppose que le vecteur paramètre est dans un compact d'un espace euclidien et que toutes ses composantes sont séparées par une distance minimale, on montre la consistance forte de l'ensemble des paramétres assez proches du minimum de variance étendue. Pour citer cet article :

\section{Introduction}

Nous considérons une généralisation de la variance intra-classe qui est considérée comme le principal critère de mesure de qualité des cartes de Kohonen (cf Kohonen [4]), bien que l'algorithme de Kohonen ne minimise pas exactement ce critère (cf Cottrell et al. [1]). La variance étendue est la somme de la variance intra-classe et d'un terme qui dépend des classes voisines. Sa minimisation permet notamment d'obtenir une classification qui respecte les relations de voisinage et qui donne lieu à des interprétations

Email address: joseph.rynkiewicz@univ-paris1.fr (Joseph Rynkiewicz). 
aisées, puisque la proximité des classes correspond à la proximité des données réelles représentées par ces classes.

Nous considérons dans toute la suite que les observations i.i.d. sont dans le compact $[0,1]^{d}$, qu'elles ont pour mesure de probabilité $P$ qui admet une densité par rapport à la mesure de Lebesque, bornée par une constante $B$. Dans la suite, on appelera "centroïde" un vecteur de $[0,1]^{d}$ qui représente une classe d'observations $\omega$.

Définition 1.1 Pour $e \in \mathbb{N}^{*}, e \leq d$, soit un ensemble fini $I \subset \mathbb{Z}^{e}$ et $\Lambda$ la fonction de voisinage définie de $I-I:=\{i-j, i, j \in I\}$ dans $[0,1]$ telle que $\Lambda(k)=\Lambda(-k)$ et $\Lambda(0)=1$.

Définition 1.2 Notons $\|$.$\| la norme euclidienne, soit$

$$
D_{I}^{\delta}:=\left\{x:=\left(x_{i}\right)_{i \in I} \in\left([0,1]^{d}\right)^{I} \text { tels que }\left\|x_{i}-x_{j}\right\| \geq \delta \text {, si } i \neq j\right\}
$$

l'ensemble des centroïdes $x_{i}$ séparés par une distance d'au moins $\delta$.

Définition 1.3 La tessellation de Voronoï $\left(C_{i}(x)\right)_{i \in I}$ est définie par

$$
C_{i}(x):=\left\{\omega \in[0,1]^{d} \text { tels que }\left\|x_{i}-\omega\right\|<\left\|x_{j}-\omega\right\| \text { si } j \neq i\right\}
$$

En cas d'ex-aequo, on assigne $\omega \in C_{i}(x)$ grâce à l'ordre lexicographique sur I. On remarquera alors que $\left(C_{i}(x)\right)_{i \in I}$ est une partition borélienne dont l'intérieur coincide avec la mosaïque de Voronoï ouverte. Réciproquement, l'indice de la tesselation de Voronoï pour une observation $\omega$ est définie par

$$
C_{x}^{-1}(\omega):=i \in I \text { tel que } \omega \in C_{i}(x)
$$

Définition 1.4 La variance étendue est : $V(x):=\frac{1}{2} \sum_{i, j \in I} \Lambda(i-j) \int_{C_{i}(x)}\left\|x_{j}-\omega\right\|^{2} d P(\omega)$

De même, lorsqu'il y a un nombre fini $n$ d'observations, on définit la variance étendue empirique :

$V_{n}(x):=\frac{1}{2 n} \sum_{i \in I} \sum_{\omega \in C_{i}(x)}\left(\sum_{j \in I} \Lambda(i-j)\left\|x_{j}-\omega\right\|^{2}\right)$

Si une observation se trouve sur un hyperplan médiateur entre deux centroïdes, tout déplacement d'un de ces deux centroïdes entrainera un saut de la variance étendue à moins que la mesure de cette observation ne soit nulle (par exemple, si la mesure ne charge pas les traces d'hyperplan). La fonction de variance étendue empirique $V_{n}(x)$ n'est donc pas continue et il n'existe pas, en général, d'ensemble de centroïdes réalisant son minimum. Cependant, si on considère les suites $x^{n}$ telles que $V_{n}\left(x^{n}\right)$ soit suffisamment proche de son minimum, on peut se demander si ces suites convergent vers l'ensemble des centroïdes minimisant la variance théorique $V(x)$. Pour cela, nous procédons selon le même schéma de démonstration que Pollard [5] et nous commençons par montrer que les fonctions de variance étendue vérifient une loi uniforme des grands nombres.

\section{Loi uniforme des grands nombres}

Soit la famille de fonctions

$$
\mathcal{G}:=\left\{g_{x}(\omega):=\sum_{j \in I} \Lambda\left(C_{x}^{-1}(\omega)-j\right)\left\|x_{j}-\omega\right\|^{2} \text { pour } x \in D_{I}^{\delta}\right\}
$$

Pour montrer la loi uniforme des grands nombres, il suffit de montrer que

$$
\sup _{x \in D_{I}^{\delta}}\left|\int g_{x}(\omega) d P_{n}(\omega)-\int g_{x}(\omega) d P(\omega)\right| \stackrel{p . s .}{\longrightarrow} 0
$$


puisque, pour toute mesure de probabilité $Q$ sur $[0,1]^{d}$ :

$$
\int g_{x}(\omega) d Q(\omega)=\int \sum_{j \in I} \Lambda\left(C_{x}^{-1}(\omega)-j\right)\left\|x_{j}-\omega\right\|^{2} d Q(\omega)=\frac{1}{2} \sum_{i, j \in I} \Lambda(i-j) \int_{C_{i}(x)}\left\|x_{j}-\omega\right\|^{2} d Q(\omega)
$$

d'après Gaenssler et Stute [3], une condition suffisante pour que l'équation (1) soit vérifiée est que : $\forall \varepsilon>0, \forall x_{0} \in D_{I}^{\delta}$ il existe un voisinage $S\left(x_{0}\right)$ de $x_{0}$ tel que

$$
\int g_{x_{0}}(\omega) d P(\omega)-\varepsilon<\int\left(\inf _{x \in S\left(x_{0}\right)} g_{x}(\omega)\right) d P(\omega) \leq \int\left(\sup _{x \in S\left(x_{0}\right)} g_{x}(\omega)\right) d P(\omega)<\int g_{x_{0}}(\omega) d P(\omega)+\varepsilon
$$

On peut d'abord prouver le résultat suivant, en utilisant une technique similaire à la preuve du lemme 11 de Fort et Pagès [2].

Lemme 2.1 Soit $x \in D_{I}^{\delta}$ et $\lambda$ la mesure de Lebesgue sur $[0,1]^{d}$. Notons $E^{c}$ le complémentaire de l'ensemble $E$ dans $[0,1]^{d}$ et $|I|$ le cardinal de l'ensemble I. Pour $0<\alpha<\frac{\delta}{2}$, soit

$U_{i}^{\alpha}(x)=\left\{\omega \in[0,1]^{d} / \exists y \in D_{I}^{\delta}, x_{j}=y_{j}\right.$ si $j \neq i$ et $\left\|x_{i}-y_{i}\right\|<\alpha$ et $\left.\omega \in C_{i}^{c}(y) \cap C_{i}(x)\right\}$

l'ensemble des $\omega$ changeant de cellule de Voronoï lorsque le centroïde $x_{i}$ se déplace d'une distance d'au plus $\alpha$. Alors

$$
\sup _{x \in D_{I}^{\delta}} \lambda\left(U_{i}^{\alpha}(x)\right)<(|I|-1)\left(\frac{2 \alpha}{\delta}+\alpha\right)(\sqrt{2})^{d-1}
$$

Considérons maintenant $x^{0} \in D_{I}^{\delta}$ et $S\left(x^{0}\right)$ un voisinage de $x^{0}$ inclus dans une boule de rayon $\alpha$, pour la distance euclidienne sur $D_{I}^{\delta}$. Soit $W\left(x^{0}\right)$ l'ensemble des $\omega$ restant dans leur cellule de Voronoï lorsque on déplace $x^{0}$ vers n'importe quel $x \in S\left(x_{0}\right)$. Pour tout $\omega \in W\left(x^{0}\right)$ on a

$$
\begin{aligned}
& \inf _{x \in S\left(x^{0}\right)} g_{x}(\omega) \geq g_{x^{0}}(\omega)-\sum_{j \in I} \Lambda\left(C_{x^{0}}^{-1}(\omega)-j\right)\left(\left\|x_{j}^{0}-\omega\right\|^{2}-\inf _{x \in S\left(x^{0}\right)}\left\|x_{j}^{0}-\omega\right\|^{2}\right) \\
& \geq g_{x_{j}^{0}}(\omega)-\sum_{j \in I}\left(\left\|x_{j}^{0}-\omega\right\|^{2}-\inf _{x \in S\left(x^{0}\right)}\left\|x_{j}^{0}-\omega\right\|^{2}\right)
\end{aligned}
$$

Pour tout $\omega \in[0,1]^{d}$, on a, pour $\alpha$ suffisamment petit, $\left(\left\|x_{j}^{0}-\omega\right\|^{2}-\inf _{x \in S\left(x^{0}\right)}\left\|x_{j}-\omega\right\|^{2}\right)<\frac{\varepsilon}{2 B|I|}$ ainsi

$$
\int_{W\left(x^{0}\right)} \sum_{j \in I}\left(\left\|x_{j}^{0}-\omega\right\|^{2}-\inf _{x \in S\left(x^{0}\right)}\left\|x_{j}-\omega\right\|^{2}\right) d P(\omega)<\frac{\varepsilon}{2} \text { et } \int_{W\left(x^{0}\right)}\left(g_{x^{0}}(\omega)-\inf _{x \in S\left(x^{0}\right)} g_{x}(\omega)\right)<\frac{\varepsilon}{2}
$$

Soit, maintenant $W\left(x^{0}\right)^{c}$, l'ensemble des $\omega$ changeant de cellule de Voronoï quand les centrö̈des vont de $x^{0}$ vers un $x \in S_{x^{0}}$. Si $\alpha<\frac{\delta}{2|I|}$, alors en déplaçant séquentiellement les composantes $x_{i}^{0}$ de $x^{0}$ vers $x_{i}$ de $x$, chaque configuration intermédaire reste dans $D_{I}^{\frac{\delta}{2}}$. Comme, pour tout $i \in I,\left\|x_{i}-\omega\right\|^{2}$ est borné par 1 sur $[0,1]^{d}$, le lemme 2.1 , assure alors que

$$
\left.\int_{W\left(x^{0}\right)^{c}} g_{x}(\omega) d P(\omega)<B|I|(|I|-1)\right)\left(\frac{4 \alpha}{\delta}+\alpha\right)(\sqrt{2})^{d-1}
$$

Finalement, si on choisit $\alpha$ suffisamment petit pour que $B|I|(|I|-1))\left(\frac{4 \alpha}{\delta}+\alpha\right)(\sqrt{2})^{d-1}<\frac{\varepsilon}{2}$, on obtient

$$
\int_{D_{I}^{\delta}} g_{x^{0}}(\omega) d P(\omega)-\varepsilon<\int_{D_{I}^{\delta}}\left(\inf _{x \in S\left(x^{0}\right)} g_{x}(\omega)\right) d P(\omega)
$$


Exactement de la même façon, pour $\alpha$ suffisamment petit, on obtient :

$$
\int_{D_{I}^{\delta}}\left(\sup _{x \in S\left(x^{0}\right)} g_{x}(\omega)\right) d P(\omega)<\int_{D_{I}^{\delta}} g_{x^{0}}(\omega) d P(\omega)+\varepsilon
$$

Ainsi, la condition suffisante pour la loi uniforme des grands nombres est vraie pour la variance étendue.

\section{Consistance}

On veut montrer la consistance des centrö̈des qui minimisent "presque" la variance étendue dans $D_{I}^{\delta}$. Soit l'ensemble des "quasi-estimateurs" de minimum de variance étendue :

$$
\bar{\chi}_{n}^{\beta}:=\left\{x \in D_{I}^{\delta} \text { tels que } V_{n}(x)<\inf _{x \in D_{I}^{\delta}} V_{n}(x)+\frac{1}{\beta(n)}\right\}
$$

avec $\beta(n)$ une fonction strictement positive tel que $\lim _{n \rightarrow+\infty} \beta(n)=\infty$. Soit $\bar{\chi}=\arg \min _{x \in D_{I}^{\delta}} V(x)$ l'ensemble qui minimise la variance étendue théorique, comme la fonction $x \longmapsto V(x)$ est continue et non constante sur $D_{I}^{\delta}$, pour tout voisinage $\mathcal{N}$ de $\bar{\chi}$, il existe $\eta(\mathcal{N})>0$ tel que

$$
\forall x \in D_{I}^{\delta} \backslash \mathcal{N}, V(x)>\min _{x \in D_{I}^{\delta}} V(x)+\eta(\mathcal{N})
$$

Pour montrer la consistance forte, il suffit de montrer que pour tout voisinage $\mathcal{N}$ de $\bar{\chi}$ on a

$$
\lim _{n \rightarrow \infty} \bar{\chi}_{n}^{\beta} \stackrel{p . s}{\subset} \mathcal{N} \Longleftrightarrow \lim _{n \rightarrow \infty} V\left(\bar{\chi}_{n}^{\beta}\right)-V(\bar{\chi}) \stackrel{p . s .}{\leq} \eta(\mathcal{N})
$$

avec $V(E)-V(F):=\sup \{V(x)-V(y)$ pour $x \in E$ et $y \in F\}$.

Par définition $V_{n}\left(\bar{\chi}_{n}^{\beta}\right) \stackrel{p . s .}{\leq} V_{n}(\bar{\chi})+\frac{1}{\beta(n)}$, de plus la loi uniforme des grands nombres assure que $\lim _{n \rightarrow \infty} V_{n}(\bar{\chi})-V(\bar{\chi}) \stackrel{p . s}{=} 0$, on obtient ainsi $\lim _{n \rightarrow \infty} V_{n}\left(\bar{\chi}_{n}^{\beta}\right) \stackrel{p . s .}{\leq} V(\bar{\chi})+\frac{\eta(\mathcal{N})}{2}$, de même on aura $\lim _{n \rightarrow \infty} V\left(\bar{\chi}_{n}^{\beta}\right)-V_{n}\left(\bar{\chi}_{n}^{\beta}\right) \stackrel{p . s .}{=} 0$ et

$$
\lim _{n \rightarrow \infty} V\left(\bar{\chi}_{n}^{\beta}\right)-\frac{\eta(\mathcal{N})}{2} \stackrel{p . s .}{<} \lim _{n \rightarrow \infty} V_{n}\left(\bar{\chi}_{n}^{\beta}\right) \stackrel{p . s .}{\leq} V(\bar{\chi})+\frac{\eta(\mathcal{N})}{2}
$$

finalement $\lim _{n \rightarrow \infty} V\left(\bar{\chi}_{n}^{\beta}\right)-V(\bar{\chi}) \stackrel{p . s .}{\leq} \eta(\mathcal{N})$ ce qui prouve la consistance forte du quasi-estimateur de minimum de variance étendue.

\section{Références}

[1] Cottrell, M., Fort, J.C. and Pagès, G., Theoretical aspects of the SOM algorithm, Neurocomputing, volume 21, p. 119-138, 1998

[2] Fort, J.C and Pagès, G., On the A.S. convergence of the Kohonen algorithm with a general neighborhood function, Ann. Appl. Prob., volume 5 :4, p.1177-1216, 1995

[3] Gaenssler, P., Stute, W., Empirical processes : A survey of results for independent and identically distributed random variables, Ann. Prob., volume $7: 2$ (1979), p.193-243

[4] Kohonen, T., Self-Organizing Maps, Springer Series in Information Sciences, Volume 30, Springer, 1995.

[5] Pollard, D., Strong consistency of k-mean clustering, Ann. Stat., volume 9 :1 (1981), p. 135-140 